\title{
The Cauchy problems for discontinuous fuzzy systems under generalized differentiability
}

\author{
Qiang Ma ${ }^{\mathrm{a}}$, Ya-Bin Shaob,*, Zeng-Tai Gong ${ }^{\mathrm{c}}$ \\ ${ }^{a}$ Network Information Management Center, Northwest University for Nationalities Lanzhou 730030, Gansu, P. R. China. \\ ${ }^{b}$ School of Science, Chongqing University of Posts and Telecommunications, Nan'an 400065, Chongqing, P. R. China. \\ ${ }^{c}$ College of Mathematics and Statistics, Northwest Normal University, Lanzhou 730070, Gansu, P. R. China.
}

Communicated by $\mathrm{X}$. Liu

\begin{abstract}
In this paper, we provide some existence theorems of generalized solutions to initial value problems for the discontinuous fuzzy differential equations and the retarded fuzzy functional differential equations by using properties of strong fuzzy Henstock integrals under strong GH-differentiability. (C)2017 All rights reserved.
\end{abstract}

Keywords: Fuzzy number, strong fuzzy Henstock integral, fuzzy differential equations, fuzzy retarded functional differential equations, fuzzy generalized solution.

2010 MSC: 47H10, 54H25.

\section{Introduction}

The Cauchy problems for fuzzy differential equations have been studied by several authors [12, 16, $17,24,25,27]$ on the metric space $\left(E^{n}, D\right)$ of normal fuzzy convex set with the distance $D$ given by the maximum of the Hausdorff distance between the corresponding level sets. In [24], the author has proved the Cauchy problem has a uniqueness result if $f$ was continuous and bounded. In [16, 17], the authors presented a uniqueness result when $f$ satisfies a Lipschitz condition. Because the metric space $\left(E^{n}, D\right)$ has a linear structure, it can be imbedded isomorphically as a cone in a Banach space. It is worth mentioning that Chen et al. [4-6] studied the initial value problems of fuzzy differential equations by using the parametric representation of fuzzy numbers and the new framework of calculus for fuzzy number valued functions established in [7]. One can see that their method was more convenient than the original method to calculate derivatives, integrals and compute numerical solutions, etc. In the very interesting paper [26], Qiu et al. introduced a metric on the quotient space of fuzzy numbers and then dealt with fuzzy mappings of a real variable whose values are equivalence classes of fuzzy numbers and gave an existence and uniqueness theorem for a solution to a fuzzy differential equation. In addition, Wang et al. [31] proved that generalized Bernstein fuzzy systems were universal approximators to a given

\footnotetext{
*Corresponding author

Email addresses: mq@xbmu .edu.cn (Qiang Ma), yb-shao@163.com (Ya-Bin Shao), yb-shao@163.com (Zeng-Tai Gong)
} 
continuous function and its high-order derivatives and the extreme learning machine method was used to tune the parameters of generalized Bernstein fuzzy system and spline fuzzy system. In 2002, Xue and $\mathrm{Fu}$ [35] established solutions to fuzzy differential equations with right-hand side functions satisfying Carathéodory conditions on a class of Lipschitz fuzzy sets.

In dynamic systems, delay is generally inevitable, that is, the velocity of the system at some instant depends on the history of the trajectory until this instant. The theory of retarded functional differential equations has been well-known when the right side function is continuous, hence Riemann integral. Hale [15] proved that the results still hold true when continuity of right function is weakened to satisfaction of a Carathéodory condition. The further step of generalization was done in [8] and [29] which applies the Henstock integrals to the study of retarded functional differential equations with finite delays and unbounded delays. Combining the fuzzy mathematics and functional differential equations, we get fuzzy functional differential equations, which have attracted the interest of many researchers [1, 22, 30]. Guo et al. [14] discussed the oscillation properties of a class of fuzzy delay differential equation of second order and provided an oscillation criterion. In [18], Khastan et al. provided sufficient conditions for the global existence of a unique (ii)-solution to an initial value problem for fuzzy functional differential equations using generalized derivative and were of broader applicability than those using Hukuhara derivative. In this paper, we extend and complement those of various authors such as [1,21,22], where the existence of generalized solution to the discontinuous fuzzy functional problem is considered by using properties of strong fuzzy Henstock integrals $[32,33]$ under strong GH-differentiability.

The paper is organized as follows. In Section 2, we give some basic concepts and preliminary results. In Section 3 and Section 4, we provide some existence theorems of generalized solutions to initial value problems for the discontinuous fuzzy differential equations and retarded fuzzy functional differential equations, respectively. Finally, in Section 5, we give some concluding remarks.

\section{Preliminaries}

Let $P_{k}\left(R^{n}\right)$ denote the family of all nonempty compact convex subsets of $R^{n}$ and define the addition and scalar multiplication in $P_{k}\left(R^{n}\right)$ as usual. Let $A$ and $B$ be two nonempty bounded subsets of $R^{n}$. The distance between $A$ and $B$ is defined by the Hausdorff metric:

$$
d_{H}(A, B)=\max \left\{\sup _{a \in A} \inf _{b \in B}\|a-b\|, \sup _{b \in B} \inf _{a \in A}\|b-a\|\right\} .
$$

Denote $E^{n}=\left\{u: R^{n} \rightarrow[0,1] \mid u\right.$ satisfies (1)-(4) below $\}$ is a fuzzy number space. where

(1) $u$ is normal, i.e., there exists an $x_{0} \in R^{\mathfrak{n}}$ such that $u\left(x_{0}\right)=1$;

(2) $u$ is fuzzy convex, i.e., $u(\lambda x+(1-\lambda) y) \geqslant \min \{u(x), u(y)\}$ for any $x, y \in R^{n}$ and $0 \leqslant \lambda \leqslant 1$;

(3) $u$ is upper semi-continuous;

(4) $[u]^{0}=\operatorname{cl}\left\{x \in R^{\mathfrak{n}} \mid u(x)>0\right\}$ is compact.

For $0<\alpha \leqslant 1$, denote $[u]^{\alpha}=\left\{x \in R^{n} \mid u(x) \geqslant \alpha\right\}$. Then from above (1)-(4), it follows that the $\alpha$-level set $[\mathrm{u}]^{\alpha} \in \mathrm{P}_{\mathrm{k}}\left(\mathrm{R}^{\mathrm{n}}\right)$ for all $0 \leqslant \alpha<1$.

Define $D: E^{n} \times E^{n} \rightarrow[0, \infty)$

$$
\mathrm{D}(\mathrm{u}, v)=\sup \left\{\mathrm{d}_{\mathrm{H}}\left([\mathrm{u}]^{\alpha},[v]^{\alpha}\right): \alpha \in[0,1]\right\},
$$

where $d$ is the Hausdorff metric defined in $P_{k}\left(R^{n}\right)$. Then it is easy to see that $D$ is a metric in $E^{n}$. Using the results in [9], we know that

(1) $\left(E^{n}, D\right)$ is a complete metric space;

(2) $\mathrm{D}(u+w, v+w)=\mathrm{D}(u, v)$ for all $u, v, w \in \mathrm{E}^{n}$;

(3) $\mathrm{D}(\lambda u, \lambda v)=|\lambda| \mathrm{D}(u, v)$ for all $u, v, w \in \mathrm{E}^{n}$ and $\lambda \in R$. 
The metric space $\left(E^{n}, D\right)$ has a linear structure, it can be imbedded isomorphically as a cone in a Banach space of function $u^{*}: I \times S^{n-1} \longrightarrow R$, where $S^{n-1}$ is the unit sphere in $R^{n}$, with an imbedding function $u^{*}=j(u)$ defined by

$$
u^{*}(r, x)=\sup _{\alpha \in[u]^{\alpha}}\langle\alpha, x>
$$

for all $<r, x>\in I \times S^{n-1}$ (see [9]).

Theorem 2.1 ([34]). There exists a real Banach space $\mathrm{X}$ such that $\mathrm{E}^{\mathrm{n}}$ can be imbedded as a convex cone $\mathrm{C}$ with vertex 0 into X. Furthermore the following conditions hold true:

(1) the imbedding $j$ is isometric;

(2) addition in $\mathrm{X}$ induces addition in $\mathrm{E}^{n}$;

(3) multiplication by nonnegative real number in $\mathrm{X}$ induces the corresponding operation in $\mathrm{E}^{\mathrm{n}}$;

(4) $\mathrm{C}-\mathrm{C}$ is dense in $\mathrm{X}$;

(5) $\mathrm{C}$ is closed.

It is well-known that the H-derivative for fuzzy-number-functions was initially introduced by Puri and Ralescu [10, 25] and it is based on the condition $(\mathrm{H})$ of sets. We note that this definition is fairly strong, because the family of fuzzy-number-valued functions H-differentiable is very restrictive. In this paper we consider a more general definition of a derivative for fuzzy-number-valued functions enlarging the class of differentiable fuzzy-number-valued functions, which has been introduced in [3].

Definition 2.2 ([3]). Let $\tilde{f}:(a, b) \rightarrow E^{n}$ and $x_{0} \in(a, b)$. We say that $\tilde{f}$ is ( $\left.\mathfrak{i}\right)$-differentiable at $x_{0}$, if there exists an element $\tilde{f}^{\prime}\left(t_{0}\right) \in E^{n}$, such that for all $h>0$ sufficiently small, there exist $\tilde{f}\left(x_{0}+h\right) \ominus_{H} \tilde{f}\left(x_{0}\right), \tilde{f}\left(x_{0}\right) \ominus_{H}$ $\tilde{f}\left(x_{0}-h\right)$ and the limits (in the metric D)

$$
\lim _{h \rightarrow 0} \frac{\tilde{f}\left(x_{0}+h\right) \ominus_{H} \tilde{f}\left(x_{0}\right)}{h}=\lim _{h \rightarrow 0} \frac{\tilde{f}\left(x_{0}\right) \ominus_{H} \tilde{f}\left(x_{0}-h\right)}{h}=\tilde{f}^{\prime}\left(x_{0}\right) .
$$

$\tilde{f}$ is (ii)-differentiable at $x_{0}$, for all $h<0$ sufficiently small, there exist $\tilde{f}\left(x_{0}+h\right) \ominus_{H} \tilde{f}\left(x_{0}\right), \tilde{f}\left(x_{0}\right) \ominus_{H}$ $\tilde{f}\left(x_{0}-h\right)$ and the limits (in the metric D)

$$
\lim _{h \rightarrow 0} \frac{\tilde{f}\left(x_{0}+h\right) \ominus_{H} \tilde{f}\left(x_{0}\right)}{h}=\lim _{h \rightarrow 0} \frac{\tilde{f}\left(x_{0}\right) \ominus_{H} \tilde{f}\left(x_{0}-h\right)}{h}=\tilde{f}^{\prime}\left(x_{0}\right) .
$$

Definition 2.3 ([20]). Let $\delta(\xi)$ be a positive real function on a closed set $[a, b]$. A division $P=\left\{\left(\xi_{i},\left[x_{i-1}, x_{i}\right]\right)\right\}$ is said to be $\delta$-fine, if the following conditions are satisfied:

(1) $a=x_{1}<x_{2}<\cdots<x_{n}=b$;

(2) $\xi_{i} \in\left[x_{i-1}, x_{i}\right] \subset\left(\xi_{i}-\delta\left(\xi_{i}\right), \xi_{i}+\delta\left(\xi_{i}\right)\right)$.

Definition 2.4 ([13]). A fuzzy-number-valued function $\tilde{f}$ will be termed additive on $[a, b]$ if for any division $T: a \leqslant x_{1} \leqslant x_{2} \leqslant \cdots \leqslant x_{n} \leqslant b$, we have $\tilde{f}\left(\left[x_{i}, x_{j}\right]\right)(1 \leqslant i<j \leqslant n)$ exists and $\tilde{f}\left(\left[x_{i}, x_{j}\right]\right)=$ $\sum_{k=i}^{j-1} \tilde{f}\left(\left[x_{k}, x_{k+1}\right]\right)$ or $\tilde{f}\left(\left[x_{j}, x_{i}\right]\right)(1 \leqslant i<j \leqslant n)$ exists and $(-1) \cdot \tilde{f}\left(\left[x_{j}, x_{i}\right]\right)=(-1) \cdot \sum_{k=i}^{j-1} \tilde{f}\left(\left[x_{k+1}, x_{k}\right]\right)$. For convenience, denote $\tilde{f}([s, t])$ by $\tilde{f}(t) \ominus_{H} \tilde{f}(s)$.

Definition 2.5 ([11, 13]). A fuzzy-number-valued function $\tilde{f}$ is said to be strong Henstock integrable on $[a, b]$ if there exists a additive fuzzy-number-valued function $\tilde{F}$ on $[a, b]$ such that for every $\varepsilon>0$ there is a function $\delta(\xi)>0$ and for any $\delta$-fine division $P=\{([u, v], \xi)\}$ of $[a, b]$, we have

$$
\sum_{i \in K_{n}} \mathrm{D}\left(\tilde{\mathrm{f}}\left(\xi_{i}\right)\left(v_{i}-u_{i}\right), \tilde{\mathrm{F}}\left(\left[u_{i}, v_{i}\right]\right)\right)+\sum_{j \in I_{n}} \mathrm{D}\left(\tilde{f}\left(\xi_{j}\right)\left(v_{j}-u_{j}\right),(-1) \cdot \tilde{F}\left(\left[u_{j}, v_{j-1}\right]\right)\right)<\varepsilon,
$$

where $K_{n}=\{i \in\{1,2, \cdot ., n\}\}$ such that $\tilde{F}\left(\left[x_{i-1}, x_{i}\right]\right)$ is a fuzzy number and $I_{n}=\{j \in\{1,2, \cdot \cdot, n\}\}$ such that $\tilde{F}\left(\left[x_{j}, x_{j-1}\right]\right)$ is a fuzzy number. We write $\tilde{f} \in \operatorname{SFH}[a, b]$. 
Definition 2.6 ([13]). A fuzzy-number-valued function $\tilde{\mathrm{F}}$ defined on $X \subset[a, b]$ is said to be $A C^{*}(X)$ if for every $\varepsilon>0$ there exists $\eta>0$ such that for every finite sequence of non-overlapping intervals $\left\{\left[a_{i}, b_{i}\right]\right\}$, satisfying $\sum_{i=1}^{n}\left|b_{i}-a_{i}\right|<\eta$ where $a_{i}, b_{i} \in X$ for all $i$ we have

$$
\sum \omega\left(\tilde{\mathrm{F}},\left[\mathrm{a}_{i}, \mathrm{~b}_{i}\right]\right)<\varepsilon,
$$

where $\omega$ denotes the oscillation of $\tilde{F}$ over $\left[a_{i}, b_{i}\right]$, i.e.,

$$
\omega\left(\tilde{F},\left[a_{i}, b_{i}\right]\right)=\sup \left\{D(\tilde{F}(y), \tilde{F}(x)) ; x, y \in\left[a_{i}, b_{i}\right]\right\} .
$$

Definition 2.7 ([13]). A fuzzy-number-valued function $\tilde{F}$ is said to be $A C G^{*}$ on $X$, if $X$ is the union of a sequence of closed sets $\left\{X_{i}\right\}$ such that on each $X_{i}, \tilde{F}$ is $A C^{*}\left(X_{i}\right)$.

For the strong fuzzy Henstock integrable we have the following theorems.

Theorem 2.8. Let $\tilde{\mathrm{f}}:[\mathrm{a}, \mathrm{b}] \rightarrow \mathrm{E}^{\mathrm{n}}$. If $\tilde{\mathrm{f}}=0$ a.e. on $[\mathrm{a}, \mathrm{b}]$, then $\tilde{\mathrm{f}}$ is SFH integrable on $[\mathrm{a}, \mathrm{b}]$ and $\int_{\mathrm{a}}^{\mathrm{b}} \tilde{\mathrm{f}}(\mathrm{t}) \mathrm{dt}=0$.

Theorem 2.9. Let $\tilde{f}:[a, b] \rightarrow E^{n}$ be SFH integrable on $[a, b]$ and let $\tilde{F}(x)=\int_{a}^{x} \tilde{f}(t) d t$ for each $x \in[a, b]$. Then

(a) the function $\tilde{\mathrm{F}}$ is continuous on $[\mathrm{a}, \mathrm{b}]$;

(b) the function $\tilde{\mathrm{F}}$ is differentiable a.e on $[\mathrm{a}, \mathrm{b}]$ and $\tilde{\mathrm{F}}^{\prime}=\mathrm{f}$;

(c) $\tilde{f}$ is measurable.

Theorem 2.10 (Controlled Convergence Theorem [13]). Suppose $\left\{\tilde{f}_{n}\right\}$ is a sequence of SFH integrable functions on $[\mathrm{a}, \mathrm{b}]$ satisfying the following conditions:

(1) $\tilde{f}_{n}(x) \rightarrow \tilde{f}(x)$ a.e. in $[\mathrm{a}, \mathrm{b}]$ as $\mathrm{n} \rightarrow \infty$;

(2) the primitives $\tilde{\mathrm{F}}_{\mathrm{n}}$ of $\tilde{\mathrm{f}}_{\mathrm{n}}$ are $\mathrm{ACG}{ }^{*}$ uniformly in $\mathrm{n}$;

(3) the primitives $\tilde{F}_{n}$ converge uniformly on $[a, b]$;

then $\tilde{f}$ is also SFH integrable on $[\mathrm{a}, \mathrm{b}]$ and

$$
\lim _{n \rightarrow \infty} \int_{a}^{b} \tilde{f}_{n}(x) d x=\int_{a}^{b} \tilde{f}(x) d x .
$$

\section{The existence of solutions for discontinuous fuzzy ordinary differential equations}

In this section, according to the idea of [19] and the operator $j$ which is the isometric embedding from $\left(E^{n}, D\right)$ onto its range in the Banach space $X$, we will deal with the Cauchy problem of discontinuous systems as following

$$
\left\{\begin{array}{l}
x^{\prime}(t)=\tilde{f}(t, x(t)) \\
x(0)=x_{0} \in E^{n}
\end{array}\right.
$$

where $E^{n}$ is a fuzzy number space, and $\tilde{f}:[0, \gamma] \times B \rightarrow E^{n}$ is strong fuzzy Henstock integrable, $B=\{x$ : $\left.\mathrm{D}\left(\mathrm{x}, \tilde{0} \leqslant \mathrm{D}\left(\mathrm{x}_{0}, \tilde{0}\right)+\mathrm{b}, \mathrm{b}>0\right)\right\}$.

Definition 3.1. Assume $x:[a, b] \rightarrow E^{n}$ is a solution of the initial value problem (3.1), if and only if $x$ is continuous and satisfied integral equation

$$
x(t)=x_{0}+\int_{t_{0}}^{t} f(s, x(s)) d s
$$

or

$$
x_{0}=x(t)+(-1) \cdot \int_{t_{0}}^{t} f(s, x(s)) d s,
$$

on some interval $\left(t_{0}, t\right) \subset R$, under the strong differentiability condition (i) or (ii), respectively. 
For any bounded subset $A$ of the Banach space $X$ we denote $\alpha(A)$ the Kuratowski measure of noncompactness of $A$, i.e., the infimum of all $\varepsilon>0$ such that there exists a finite covering of $A$ by sets of diameter less than $\varepsilon$. For the properties of $\alpha$ we refer to [2] for example.

Lemma 3.2 ([2]). Let $\mathrm{H} \subset \mathrm{C}\left(\mathrm{I}_{\gamma}, \mathrm{X}\right)$ be a family of strong equicontinuous functions. Then

$$
\alpha(\mathrm{H})=\sup _{\mathrm{t} \in \mathrm{I}_{\gamma}} \alpha(\mathrm{H}(\mathrm{t}))=\alpha\left(\mathrm{H}\left(\mathrm{I}_{\gamma}\right)\right),
$$

where $\alpha(\mathrm{H})$ denote the Kuratowski measure of non-compactness in $\mathrm{C}\left(\mathrm{I}_{\gamma}, \mathrm{X}\right)$ and the function $\mathrm{t} \rightarrow \alpha(\mathrm{H}(\mathrm{t}))$ is continuous.

Let $\mathrm{C}\left(\mathrm{x}_{0}, \gamma\right)=\left\{x \in C\left(\mathrm{I}_{\gamma}\right): x(0)=x_{0}, \mathrm{D}(x, \tilde{0}) \leqslant \mathrm{D}\left(\mathrm{x}_{0}, \tilde{0}\right)+\mathrm{b}\right\}$ (b and $\gamma$ are some positive numbers). Obviously, the fuzzy set $\mathrm{C}\left(\mathrm{x}_{0}, \gamma\right)$ is closed and convex.

Let $\tilde{F}_{x}$ be defined by $\tilde{F}_{x}(t)=x_{0}+\int_{0}^{t} \tilde{f}(s, x(s)) d s$ or $\tilde{F}_{x}(t)=x_{0}+(-1) \cdot \int_{0}^{t} \tilde{f}(s, x(s)) d s$, for $t \in I_{\gamma}$ and $x \in \mathrm{C}\left(\mathrm{x}_{0}, \gamma\right)$ where the integral is in the sense of SFH.

Definition 3.3. A fuzzy-number-valued function $\tilde{f}: \mathrm{I}_{\gamma} \times \mathrm{E}^{\mathrm{n}}$ is a Carathéodory function, if

(1) $\tilde{f}$ is measurable for any $x \in E^{n}$;

(2) $\tilde{f}$ is continuous for any $t \in I_{\gamma}$.

Lemma 3.4. Let $\mathrm{V}$ be equicontinuous bounded set in $\mathrm{C}\left(\mathrm{I}_{\gamma}, \mathrm{E}^{\mathrm{n}}\right)$, $\tilde{\mathrm{f}}$ be a Carathédory function and $\tilde{\mathrm{f}}(\cdot, \mathrm{x}(\cdot))$ be a SFH integrable function for each $x \in V$. Let $\tilde{\mathrm{F}}=\left\{\tilde{\mathrm{F}}_{x}: x \in \mathrm{C}\left(\mathrm{x}_{0}, \gamma\right)\right\}$ be equicontinuous and uniformly $\mathrm{ACG}^{*}$ on $\mathrm{I}_{\gamma}$. Then

$$
\alpha\left(j \circ \int_{0}^{t} \tilde{f}(s, V(s)) d s\right) \leqslant \int_{0}^{t} \alpha(j \circ \tilde{f}(s, V(s))) d s,
$$

whenever $\alpha(j \circ \tilde{f}(s, V(s))) \leqslant \varphi(s)$, for $s \in I_{\gamma}$ a.e., $\varphi(s)$ is a Lebesgue integrable function, and

$$
\int_{0}^{t} \tilde{f}(s, V(s)) d s=\left\{\int_{0}^{t} \tilde{f}(s, x(s)) d s, x(s) \in V(s)\right\}
$$

Theorem 3.5 ([23]). Let $\mathrm{D}$ be a closed convex subset of $\mathrm{X}$, and let $\mathrm{F}$ be a continuous function from $\mathrm{D}$ into itself. If for $\mathrm{x} \in \mathrm{D}$ the implication

$$
\bar{V}=\operatorname{con}(\{x\} \cup F(V)) \Rightarrow V,
$$

is relatively compact, then $\mathrm{F}$ has a fixed point.

Definition 3.6. A nonnegative function $(t, r) \rightarrow h(t, r)$ is a Kamke function on $I \times R^{+}$, if

(1) $h(t, r)$ satisfies the Carathéodory conditions;

(2) $h(t, 0)=0$ and the function identically equal to zero is the unique continuous solution of the equation $u(t)=\int_{0}^{t} h(s, u(s)) d s$, for $t \in I$ satisfying the condition $u(0)=0$.

Next, we give the main results for this section.

Theorem 3.7. If for each continuous function $\mathrm{x}: \mathrm{I}_{\gamma} \rightarrow \mathrm{E}^{\mathrm{n}}, \tilde{\mathrm{f}}(\cdot, x(\cdot))$ is SFH integrable, $\tilde{\mathrm{f}}$ is a Carathéodory function and

$$
\alpha(j \circ \tilde{f}(t, X)) \leqslant h(t, \alpha(j \circ X)),
$$

for each bounded subset $\mathrm{X} \subset \mathrm{E}^{\mathrm{n}}$, where $\mathrm{h}$ is a Kamke function. Let $\mathrm{F}=\left\{\tilde{\mathrm{F}}_{\mathrm{x}}: \mathrm{x} \in \mathrm{C}\left(\mathrm{x}_{0}, \gamma\right)\right\}$ and $\mathrm{F}$ is equicontinuous and uniformly $\mathrm{ACG}^{*}$ on $\mathrm{I}_{\gamma}$. Then there exists a solution of the problem (3.1) on $\mathrm{I}_{\beta}$ for some $0<\beta \leqslant \gamma$. 
Proof. By equicontinuous of $F$, there exist a number $\beta$, and $0<\beta \leqslant \gamma$ such that

$$
D\left(\int_{0}^{t} \tilde{f}(s, x(s)) d s, \tilde{0}\right) \leqslant b,
$$

for $t \in I_{\beta}$ and $x \in C\left(x_{0}, \alpha\right)$. By the assumption, the operator $\tilde{F}_{x}$ is well-defined and maps $C\left(x_{0}, \beta\right)$ into $C\left(x_{0}, \beta\right)$. Using Theorem 2.10 for the SFH integral we deduce that $\tilde{F}$ is continuous.

Suppose that $\bar{V}=\operatorname{con}(\{x\}) \cup F(V)$ for some bounded $V \subset C\left(x_{0}, \beta\right)$. We will prove that $V$ is relatively compact, thus (3.2) is satisfied.

In fact, $F(V)$ is equicontinuous, the function $v(t) \rightarrow \alpha(j \circ V(t))$ is continuous on $I_{\beta}$, and $F(V(t))=$ $\left\{x_{0}+\int_{0}^{t} \tilde{f}(s, x(s)) d s, x(s) \in V(s)\right\}$ or $F(V(t))=\left\{x_{0}+(-1) \cdot \int_{0}^{t} \tilde{f}(s, x(s)) d s, x(s) \in V(s)\right\}$.

By Lemma 3.4 and (3.3), we have

$$
\begin{aligned}
\alpha(j \circ F(V(t))) & \leqslant \alpha\left(j \circ\left\{\int_{0}^{t} \tilde{f}(s, x(s)) d s, \chi(s) \in V(s)\right\}\right) \\
& \leqslant \int_{0}^{t} \alpha(j \circ \tilde{f}(s, V(s))) d s \\
& \leqslant \int_{0}^{t} h(s, \alpha(j \circ V(s))) d s .
\end{aligned}
$$

Since $\bar{V}=\operatorname{con}(\{x\}) \cup F(V)$, by the property of measure of noncompactness we have $\alpha(j \circ V(t)) \leqslant$ $\alpha(j \circ F(V(t)))$ and

$$
v(t)=\alpha(j \circ V(t)) \leqslant \int_{0}^{t} h(s, v(s)) d s .
$$

Hence, we have $v(t)=\alpha(j \circ V(t))$. By Lemma 3.2, $V$ is relatively compact. So, by Theorem 3.5, $V$ has a fixed point which is a solution of (3.1).

Now, we give the theorem on the existence of solution of the fuzzy differential inclusion

$$
\left\{\begin{array}{l}
x^{\prime}(t) \in \tilde{F}(t, x(t)) \\
x(0)=x_{0} \in E^{n}
\end{array}\right.
$$

in a fuzzy number space, where the multifunction $\tilde{\mathrm{F}}: \mathrm{I}_{\gamma} \times \mathrm{E}^{\mathrm{n}} \rightarrow \mathrm{E}^{\mathrm{n}}$ is such that $\tilde{\mathrm{f}}(\cdot, x(\cdot))$ has strong fuzzy Henstock selection $v(\cdot)$ for each $x \in \mathrm{C}\left(\mathrm{x}_{0}, \mathrm{I}_{\gamma}\right)$.

Definition 3.8. The set $\left\{\int_{0}^{\gamma} \tilde{\mathrm{f}}(\mathrm{s}, \chi(\mathrm{s})) \mathrm{d} s: \tilde{\mathrm{f}}(\cdot, \chi(\cdot)) \in \tilde{\mathrm{F}}(\cdot, \chi(\cdot))\right.$; $\mathrm{f}$ is a SFH integrable $\}$ is called SFH integral of the multifunction $\tilde{\mathrm{F}}$ on $\mathrm{I}_{\gamma}$.

Theorem 3.9. Let the multifunction $\tilde{\mathrm{F}}$ has a SFH selection $\tilde{\mathrm{f}}(\cdot, x(\cdot))$ for any $\mathrm{x} \in \mathrm{C}\left(\mathrm{x}_{0}, \alpha\right)$ such that $\tilde{\mathrm{f}}$ is a Carathéodory function and define $\tilde{\mathrm{G}}_{x}(\mathrm{t})$ as

$$
\tilde{\mathrm{G}}_{x}(\mathrm{t})=x_{0}+\int_{0}^{\mathrm{t}} \tilde{\mathrm{F}}(\mathrm{s}, x(\mathrm{~s})) \mathrm{d} s
$$

or

$$
\tilde{\mathrm{G}}_{x}(\mathrm{t})=x_{0}+(-1) \cdot \int_{0}^{\mathrm{t}} \tilde{\mathrm{F}}(\mathrm{s}, x(\mathrm{~s})) \mathrm{d} \mathrm{s}
$$

for $\mathrm{t} \in \mathrm{I}$ and $\mathrm{x} \in \mathrm{C}\left(\mathrm{x}_{0} . \alpha\right)$ and

$$
\alpha(j \circ \tilde{F}(t, X)) \leqslant h(t, \alpha(j \circ X)),
$$

for each bounded subset $\mathrm{X} \subset \mathrm{E}^{\mathrm{n}}$, where $\mathrm{h}$ is a Kamke function. Let $\mathrm{G}=\left\{\tilde{\mathrm{G}}_{x}: x \in \mathrm{C}\left(\mathrm{x}_{0}, \alpha\right)\right\}$ and $\mathrm{G}$ is equicontinuous and uniformly ACG* on $\mathrm{I}_{\alpha}$. Then there exists a solution of the problem (3.4) on $\mathrm{I}_{\beta}$ for some $0<\beta \leqslant \gamma$.

Proof. It is easy to see that $\tilde{f}$ satisfies the conditions of Theorem 3.7. It is clear that every solution of (3.1) is a solution of (3.4). 


\section{The existence of solutions for discontinuous fuzzy retarded functional differential equations}

In this section, using properties of the strong fuzzy Henstock integral and corresponding controlled convergence theorems, we prove existence theorems for the retarded functional equation in a fuzzy number space.

Let $r, \alpha$ be nonnegative real numbers, $I_{\alpha}=[0, \alpha]$ and let $E^{n}$ be a fuzzy number space. Suppose that $x(t)$ is fuzzy-number-valued function defined on $[-r, \alpha]$. For any $\alpha \in I_{\alpha}$, the function $x_{t}$ is defined as $x_{t}(\theta)=x(t+\theta)$, where $-r \leqslant \theta \leqslant 0$.

Let $\tilde{f}: I_{\alpha} \times C\left([-r, 0], E^{n}\right) \rightarrow E^{n}$, and

$$
\left\{\begin{array}{l}
x^{\prime}(t)=\tilde{f}\left(t, x_{t}\right), \\
x(\theta)=\varphi(\theta), \quad-r \leqslant \theta \leqslant 0,
\end{array}\right.
$$

where the integral is taken in the sense of strong fuzzy Henstock integral and the derivative $x^{\prime}$ is considered in the sense of GH-derivative.

Let $\mathrm{SFH}[u, v]$ denote the space of fuzzy-number-valued functions of strong fuzzy Henstock integrable on $[u, v]$. We consider the metric $H(\cdot, \cdot)$ on $S F H[u, v]$ defined by

$$
\mathrm{H}(\phi(\mathrm{t}), \psi(\mathrm{t}))=\sup _{\mathrm{t} \in[\mathrm{u}, v]} \mathrm{D}(\Phi(\mathrm{t}), \Psi(\mathrm{t})),
$$

where $\Phi(t)=\int_{u}^{t} \phi(s) d s$ and $\Psi(t)=\int_{u}^{t} \psi(s) d s$, for any $\phi(t), \psi(t) \in \operatorname{SFH}[u, v]$.

Let $\varphi$ be some specified function fixed in $\operatorname{SFH}[-r, 0]$. The set $\Omega_{b}$ and $R_{\alpha b}$ are defined as follows:

$$
\Omega_{\mathrm{b}}=\{x \in \mathrm{SFH}[-\mathrm{r}, 0]: \mathrm{H}(x, \varphi) \leqslant \mathrm{b}\}, \quad \mathrm{R}_{\alpha \mathrm{b}}=\mathrm{I}_{\alpha} \times \Omega_{\mathrm{b}},
$$

where $\alpha, b$ are positive numbers.

Definition 4.1 ([28]). A nonnegative real-valued function $h(t, r)$ defined on $I_{\alpha} \times \mathbb{R}^{+}$is a Kamke function if $h$ satisfies the Carathéodory conditions, $h(t, 0)=0$ and the function identically equal to zero is the unique continuous solution of the equation $u(t)=\int_{0}^{t} h\left(s, u_{s}\right) d s$, for $t \in I_{\alpha}$ satisfying the condition $u(0)=0$, where $u_{s}=u(t+s)$.

Definition 4.2 ([35]). A fuzzy-number-valued function $\tilde{f}: R_{\alpha b} \rightarrow E^{n}$ is said to be Carathéodory function on $R_{\alpha b}$, if for each $x \in \Omega_{b}, \tilde{f}(t, x)$ is measurable in $t$ and for almost all $t \in I_{\alpha}, \tilde{f}(t, x)$ is continuous with respect to $x$.

Theorem 4.3. A fuzzy-number-valued function $\mathrm{x}(\mathrm{t})$ is called a generalized (i)-solution (or generalized (ii)-solution) of problem (4.1) if and only if it satisfies the following integral equations

$$
\left\{\begin{array}{l}
x_{0}=\varphi, \\
x(t)=\varphi(0)+\int_{0}^{t} \tilde{f}\left(s, x_{s}\right) d s, \quad t \in I_{\alpha},
\end{array}\right.
$$

or

$$
\left\{\begin{array}{l}
x_{0}=\varphi, \\
x(t)=\varphi(0) \ominus_{H}\left(-\int_{0}^{t} \tilde{f}\left(s, x_{s}\right) d s\right), \quad t \in I_{\alpha},
\end{array}\right.
$$

respectively.

We now define an auxiliary function $\hat{x}$ : if $x$ is defined on $\mathrm{I}_{\beta}(0<\beta<\alpha)$ with $x(0)=\varphi(0)$, the function $\hat{x}$ is defined as follows:

$$
\hat{x}=\left\{\begin{array}{l}
x(t), \quad t \in(0, \beta), \\
x(t)=\varphi(t), \quad t \in(-r, 0) .
\end{array}\right.
$$

Theorem 4.4. The set $\mathcal{A}(\varphi, \alpha) \subset \mathrm{C}\left(\mathrm{I}_{\alpha}, \mathrm{E}^{\mathrm{n}}\right)$ is defined by

$$
\mathcal{A}(\varphi, \alpha)=\left\{x \in \mathrm{C}\left(\mathrm{I}_{\alpha}, \mathrm{E}^{\mathfrak{n}}\right): x(0)=\varphi(0), \mathrm{D}(\chi, \tilde{0}) \leqslant \mathrm{b}+\mathrm{D}(\varphi(0), \tilde{0}), \hat{x} \in \Omega_{\mathrm{b}}\right\} .
$$

Then $\mathcal{A}(\varphi, \alpha)$ is bounded, closed and convex. 
We define the integral operator as follows:

or

$$
\tilde{F_{x}}(t)=x_{0}+\int_{0}^{t} \tilde{f}\left(s, \hat{x}_{s}\right) d s
$$

$$
\tilde{\mathrm{F}_{x}}(\mathrm{t})=\mathrm{x}_{0} \ominus_{\mathrm{H}}\left(-\int_{0}^{\mathrm{t}} \tilde{\mathrm{f}}\left(\mathrm{s}, \hat{\mathrm{x}}_{\mathrm{s}}\right) \mathrm{d} \mathrm{s}\right),
$$

for $\mathrm{t} \in \mathrm{I}_{\alpha}$ and $x \in \mathcal{A}(\varphi, \alpha)$ where the integral is taken in the sense of strong fuzzy Henstock integral and the derivative $x^{\prime}$ is considered in the sense of GH-derivative.

Theorem 4.5. Let $\mathrm{E}^{\mathrm{n}}$ be a fuzzy number space and $\mathrm{V}$ is a set of strong fuzzy Henstock integrable functions. Let

$$
\mathrm{F}=\left\{\int_{0}^{\mathrm{t}} \mathrm{x}(\mathrm{s}) \mathrm{d} \mathrm{s}, \mathrm{x} \in \mathrm{V}, \mathrm{t} \in \mathrm{I}_{\alpha}\right\},
$$

be a equi-continuous, equi-bounded and strong uniformly $\mathrm{AC}^{\nabla}$ on $\mathrm{I}_{\alpha}$. Then

$$
\mathcal{K}\left(\int_{0}^{\mathrm{t}} \mathbf{j} \circ \mathrm{V}(\mathrm{s}) \mathrm{d} \mathrm{s}\right) \leqslant \int_{0}^{\mathrm{t}} \mathcal{K}(\mathbf{j} \circ \mathrm{V}(\mathrm{s}) \mathrm{d} \mathrm{s}),
$$

whenever $\mathcal{K}(\mathrm{j} \circ \mathrm{V}(\mathrm{s})) \leqslant \mathrm{h}(\mathrm{s})$, for $\mathrm{s} \in \mathrm{I}_{\alpha}$ a.e., $\mathrm{h}$ is a Henstock integrable function and $\mathcal{K}$ denotes the Kuratowski's measure of non-compactness.

Proof. Since $x(s)$ is strong fuzzy Henstock integrable, for each $\varepsilon=\frac{1}{m}>0$ there exists a function $\delta_{m}(x)>0$ such that for any $\delta_{m}$-fine division $\left\{\left(\xi_{i},\left[u_{i}, v_{i}\right]\right)\right\}$, we have

$$
\begin{aligned}
\mathrm{D}\left(\sum x\left(\xi_{i}\right)\left(v_{i}-u_{i}\right), \int_{0}^{t} x(s) d s\right) & =\sup \left\{d_{H}\left(\left[\sum x\left(\xi_{i}\right)\left(v_{i}-u_{i}\right)\right]^{\alpha},\left[\int_{0}^{t} x(s) d s\right]^{\alpha}\right)\right\} \\
& \leqslant\left|d_{H}\left(\sum x\left(\xi_{i}\right)\left(v_{i}-u_{i}\right), j \circ V\right)-\int_{0}^{t}\left(d_{H}[x(s)]^{\alpha}, j \circ V\right) d s\right| \\
& \leqslant\left|\sum d_{H}\left(x\left(\xi_{i}\right), j \circ V\right)\left(v_{i}-u_{i}\right)-\int_{0}^{t} d_{H}\left([x(s)]^{\alpha}, j \circ V\right) d s\right| \\
& <\varepsilon_{m} .
\end{aligned}
$$

Hence, by taking $m \rightarrow \infty$ we have

$$
d_{H}\left(\left[\int_{0}^{t} x(s) d s\right]^{\alpha}, j \circ V\right) \leqslant(H) \int_{0}^{t} d_{H}\left([x(s)]^{\alpha}, j \circ V\right) d s .
$$

In addition, let $\int_{0}^{t} j \circ V(s) d s=\left\{j \circ \int_{0}^{t} x_{m}(s) d s: m=1,2, \cdots\right\}$. Since the function $d_{H}\left(\left[x_{m}(t)\right]^{\alpha}, j \circ V\right)$ is measurable on $I_{\alpha}$, we have $\mathcal{K}(j \circ V(t))=\lim _{m \rightarrow \infty} d_{H}\left(\left[x_{m}(t)\right]^{\alpha}, j \circ H_{F}\right)$ is measurable on $I_{\alpha}$ too. By (4.2) we have

$$
d_{H}\left(\left[\int_{0}^{t} x_{m}(s) d s\right]^{\alpha}, j \circ V\right) \leqslant(H) \int_{0}^{t} d_{H}\left(\left[x_{m}(s)\right]^{\alpha}, j \circ V\right) d s
$$

Hence, by Henstock's theorems monotone and dominated convergence of real valued functions, we have

$$
\begin{aligned}
\left.\lim _{m \rightarrow \infty} d_{H}\left(\left[\int_{0}^{t} x(s) d s\right]^{\alpha}, j \circ V\right]\right) & \leqslant \lim _{m \rightarrow \infty} \int_{0}^{t} d_{H}\left(\left[x_{m}(s)\right]^{\alpha}, j \circ V\right) d s \\
& \leqslant \int_{0}^{t} \lim _{m \rightarrow \infty} d_{H}\left(\left[x_{m}(s)\right]^{\alpha}, j \circ V\right) d s .
\end{aligned}
$$

So,

$$
\mathcal{K}\left(\int_{0}^{t} j \circ V(s) d s\right) \leqslant \int_{0}^{t} \mathcal{K}(j \circ V(s) d s),
$$

for $t \in I_{\alpha}$.

Now, we present the main outcomes in this section. 
Theorem 4.6. Let $\varphi$ be some specified function fixed in $\mathrm{SFH}[-\mathrm{r}, 0]$. Suppose that $\tilde{\mathrm{f}}\left(\mathrm{t}, \mathrm{x}_{\mathrm{t}}\right)$ is strong fuzzy Henstock integrable and a Carathéodory function defined on $\mathrm{R}_{\alpha \mathrm{b}}$ and

$$
\mathcal{K}(j \circ \tilde{f}(t, X)) \leqslant h(t, \mathcal{K}(j \circ X)),
$$

for each bounded subset $\mathrm{X} \subset \mathrm{C}\left(\mathrm{I}_{\alpha}, \mathbb{R}_{\mathrm{F}}\right)$, where $\mathrm{h}$ is a Kamke function. Let $\mathrm{F}=\left\{\tilde{\mathrm{F}}_{\mathrm{x}}: x \in \mathcal{A}(\varphi, \alpha)\right\}$ and suppose that $\mathrm{F}$ is equi-continuous, equi-bounded and uniformly strong $A \mathrm{C}^{\nabla}$ on $\mathrm{I}_{\alpha}$. Then there exists a generalized (i)-solution (or generalized (ii)-solution) of problem (4.2) on $\mathrm{I}_{\beta}$ for some $0<\beta \leqslant \alpha$ with initial function $\varphi$.

Proof. We only prove case of (i)-GH-differentiable. By equi-continuous of $F$, there exists some $\beta, 0<\beta \leqslant \alpha$ such that

$$
\mathrm{D}\left(\int_{0}^{\mathrm{t}} \tilde{\mathrm{f}}\left(\mathrm{s}, \hat{x}_{\mathrm{s}}\right) \mathrm{d} \mathrm{s}, \tilde{0}\right) \leqslant \mathrm{b}, \quad \text { and } \quad \mathrm{D}\left(\int_{-\tau}^{\tau} \varphi(0) \mathrm{d} s, \int_{-\tau}^{\tau} \varphi(\mathrm{s}) \mathrm{d} s\right)<\mathrm{k},
$$

and

$$
\mathrm{D}\left(\int_{-\tau}^{\tau} \int_{0}^{t+s} \tilde{f}\left(p, \hat{x}_{p}\right) \mathrm{dpds}, \tilde{0}\right)<l
$$

and $x \in \mathcal{A}(\varphi, \beta)$.

Since the integral operator $\tilde{F}_{x}$ is well-defined, in fact

$$
D\left(\varphi(0)+\int_{0}^{t} \tilde{f}\left(s, \hat{x}_{s}\right) d s, \tilde{0}\right) \leqslant D(\varphi(0), \tilde{0})+D\left(\int_{0}^{t} \tilde{f}\left(s, \hat{x}_{s}\right) d s, \tilde{0}\right)=D(\varphi(0), \tilde{0})+b,
$$

and

$$
\begin{aligned}
& \mathrm{H}\left(\hat{\mathrm{F}}_{x_{\mathrm{t}}}, \varphi\right)=\sup _{\tau \in(-\mathrm{r}, 0)} \mathrm{D}\left(\int_{-r}^{\tau} \hat{\tilde{F}}_{x_{\mathrm{t}}}(s) \mathrm{d} s, \int_{-r}^{\tau} \varphi(s) \mathrm{d} s\right) \\
& =\sup _{\tau \in(-r, 0)} D\left(\int_{-r}^{\tau} \hat{\tilde{F}}_{x}(s+t) d s, \int_{-r}^{\tau} \varphi(s) d s\right) \\
& =\sup _{\tau \in(-r, 0)} D\left(\int_{-r}^{\tau}\left[\varphi(0)+\int_{0}^{t+s} \tilde{f}\left(p, \hat{x}_{p}\right) d p\right] d s, \int_{-r}^{\tau} \varphi(s) d s\right) \\
& \leqslant \sup _{\tau \in(-r, 0)} \mathrm{D}\left(\int_{-r}^{\tau} \varphi(0) \mathrm{d} s, \int_{-\tau}^{\tau} \varphi(s) \mathrm{d} s\right) \\
& +\sup _{\tau \in(-r, 0)} D\left(\int_{-r}^{\tau} \int_{0}^{t+s} \tilde{f}\left(p, \hat{x}_{p}\right) d p d s, \tilde{0}\right) \\
& \leqslant k+l=b \text {. }
\end{aligned}
$$

Let $\overline{j \circ V}=\overline{\operatorname{conv}}\{j \circ(x \cup F(V))\}$ for $V \subset \mathcal{A}(\varphi, \alpha)$. Next, we will prove that $j \circ V$ is relatively compact.

In fact, we put

$$
\left\{\int_{0}^{t} \tilde{f}\left(s, \hat{x}_{s}\right) \mathrm{d} s, x \in V\right\}=\int_{0}^{t} \tilde{f}\left(s, V_{s}\right) d s
$$

where $\mathrm{V}_{\mathrm{s}}=\{\hat{x}: x \in \mathrm{V}\}$ and

$$
F(V(t))=\varphi(0)+\int_{0}^{t} \tilde{f}\left(s, \hat{x}_{s}\right) d s
$$

By Theorem 4.5 and (4.3) we have

$$
\begin{aligned}
\mathcal{K}(j \circ \mathrm{F}(\mathrm{V}(\mathrm{t}))) & =\mathcal{K}\left[j \circ\left(\varphi(0)+\int_{0}^{\mathrm{t}} \tilde{\mathrm{f}}\left(\mathrm{s}, \mathrm{V}_{\mathrm{s}}\right) \mathrm{d} \mathrm{s}\right)\right] \\
& \leqslant \int_{0}^{\mathrm{t}} \mathcal{K}\left[j \circ\left(\tilde{\mathrm{f}}\left(\mathrm{s}, \mathrm{V}_{\mathrm{s}}\right)\right) \mathrm{d} \mathrm{s}\right] \\
& \leqslant \int_{0}^{\mathrm{t}} \mathrm{h}(\mathrm{s}, \mathcal{K}(j \circ \mathrm{V}(\mathrm{s})) \mathrm{d} s .
\end{aligned}
$$

By the properties of the Kuratowski measure of non-compactness, we have $\mathcal{K}(j \circ V(t)) \leqslant \mathcal{K}(j \circ F(V(t)))$ 
and by differential inequality as follows

$$
v(t)=\mathcal{K}(j \circ V(t)) \leqslant \int_{0}^{t} h(s, \mathcal{K}(v(s))) d s
$$

we have $v(t)=0$. By Lemma $3.2 \mathrm{j} \circ \mathrm{V}$ is relatively compact. Therefore by Theorem 3.5 and Theorem 4.3, the problem (4.1) has a generalized (i)-solution. The proof is completed.

Example 4.7. Let $\alpha=r=1$ and define a fuzzy-number-valued function $\tilde{f}\left(t, x_{t}\right)$ as

$$
\tilde{f}\left(t, x_{t}\right)=x_{\mathfrak{t}}(-1)+\tilde{F}^{\prime}(t), \quad 0 \leqslant t \leqslant 1,
$$

where " $"$ " is (i)-GH-differentiability.

Let $\varphi$ be some initial function continuous on $[-1,0]$. The problem $(4.1)$ is

$$
x^{\prime}(t)=x_{t}(-1)+\tilde{F}^{\prime}(t)=x(t-1)+\tilde{F}^{\prime}(t),
$$

subject to the condition that $x(\theta)=\varphi(\theta)$ for $\theta \in[-1,0]$. As $t \in[-1,0]$, problem (4.1) indicates

$$
\begin{aligned}
& x^{\prime}=\varphi(t-1)+\tilde{F}^{\prime}(t) . \\
& \chi(t)=\int_{0}^{t} \varphi(s-1) d s+\int_{0}^{t} \tilde{F}^{\prime}(s) d s+\varphi(0) .
\end{aligned}
$$

Now the function $\tilde{F}^{\prime}(t)$ is not Kaleva integrable on $[0,1]$ but it is strong fuzzy Henstock integrable here. Then the problem (4.1) has a generalized (i)-solution

$$
\chi(t)=\int_{0}^{t} \varphi(s-1) \mathrm{d} s+\int_{0}^{t} \tilde{\mathrm{F}}^{\prime}(\mathrm{s}) \mathrm{d} s+\varphi(0) .
$$

\section{Conclusions}

The major contribution of this paper is to study the problems of existence of generalized solutions to discontinuous fuzzy systems such as with right-hand function is strong fuzzy Henstock integral under generalized differentiability. Some known results of fuzzy differential equations and fuzzy delay functional differential equations are extended, which might be helpful in the analysis of dynamic systems with uncertainties.

\section{Acknowledgment}

The authors thank the National Nature Science Foundation of China (Grant No. 11161041, No. 61472056 and No. 11461062), Chongqing Municipal Science and technology project (cstc2015jcyjA00015) and the PhD Research Startup Foundation of Chongqing University of Posts and Telecommunications (No. A2014-90, A2016-13).

\section{References}

[1] P. Balasubramaniam, S. Muralisankar, Existence and uniqueness of a fuzzy solution for the nonlinear fuzzy neutral functional differential equation, Comput. Math. Appl., 42 (2001), 961-967. 1

[2] J. Banaś, K. Goebel, Measures of noncompactness in Banach spaces, Lecture Notes in Pure and Applied Mathematics, Marcel Dekker, Inc., New York, (1980). 3, 3.2

[3] B. Bede, S. G. Gal, Generalizations of the differentiability of fuzzy-number-valued functions with applications to fuzzy differential equations, Fuzzy Sets and Systems, 151 (2005), 581-599. 2, 2.2

[4] M.-H. Chen, C.-S. Han, Some topological properties of solutions to fuzzy differential systems, Inform. Sci., 197 (2012), 207-214. 1 
[5] M.-H. Chen, C.-S. Han, Periodic behavior of semi-linear uncertain dynamical systems, Fuzzy Sets and Systems, 230 (2013), 82-91.

[6] M.-H. Chen, D.-H. Li, X.-P. Xue, Periodic problems of first order uncertain dynamical systems, Fuzzy Sets and Systems, 162 (2011), 67-78. 1

[7] M.-H. Chen, C.-X. Wu, X.-P. Xue, G.-Q. Liu, On fuzzy boundary value problems, Inform. Sci., 178 (2008), $1877-1892$. 1

[8] T. S. Chew, W. van-Brunt, G. C. Wake, On retarded functional-differential equations and Henstock-Kurzweil integrals, Differential Integral Equations, 9 (1996), 569-580. 1

[9] P. Diamond, P. Kloeden, Metric spaces of fuzzy sets, Theory and applications, World Scientific Publishing Co., Inc., River Edge, NJ, (1994). 2

[10] R. Goetschel, W. Voxman, Elementary fuzzy calculus, Fuzzy Sets and Systems, 18 (1986), 31-43. 2

[11] Z.-T. Gong, On the problem of characterizing derivatives for the fuzzy-valued functions, II, Almost everywhere differentiability and strong Henstock integral, Fuzzy Sets and Systems, 145 (2004), 381-393. 2.5

[12] Z.-T. Gong, Y.-B. Shao, Global existence and uniqueness of solutions for fuzzy differential equations under dissipative-type conditions, Comput. Math. Appl., 56 (2008), 2716-2723. 1

[13] Z.-T. Gong, Y.-B. Shao, The controlled convergence theorems for the strong Henstock integrals of fuzzy-number-valued functions, Fuzzy Sets and Systems, 160 (2009), 1528-1546. 2.4, 2.5, 2.6, 2.7, 2.10

[14] M.-S. Guo, X.-Y. Peng, Y.-Q. Xu, Oscillation property for fuzzy delay differential equations, Fuzzy Sets and Systems, 200 (2012), 25-35. 1

[15] J. Hale, Theory of functional differential equations, Second edition, Applied Mathematical Sciences, Springer-Verlag, New York-Heidelberg, (1997). 1

[16] O. Kaleva, Fuzzy differential equations, Fuzzy Sets and Systems, 24 (1987), 301-319. 1

[17] O. Kaleva, The Cauchy problem for fuzzy differential equations, Fuzzy Sets and Systems, 35 (1990), 389-396. 1

[18] A. Khastan, J. J. Nieto, R. Rodríguez-López, Fuzzy delay differential equations under generalized differentiability, Inform. Sci., 275 (2014), 145-167. 1

[19] I. Kubiaczyk, A. Sikorska, Differential equations in Banach space and Henstock-Kurzweil integrals, Discuss. Math. Differential Incl., 19 (1999), 35-43. 3

[20] P. Y. Lee, Lanzhou lectures on Henstock integration, Series in Real Analysis, World Scientific Publishing Co., Inc., Teaneck, NJ, (1989). 2.3

[21] V. Lupulescu, U. Abbas, Fuzzy delay differential equations, Fuzzy Optim. Decis. Mak., 11 (2012), 99-111. 1

[22] C. Min, N.-J. Huang, L.-H. Zhang, Existence of local and global solutions of fuzzy delay differential inclusions, Adv. Difference Equ., 2014 (2014), 14 pages. 1

[23] H. Mönch, Boundary value problems for nonlinear ordinary differential equations of second order in Banach spaces, Nonlinear Anal., 4 (1980), 985-999. 3.5

[24] J. J. Nieto, The Cauchy problem for continuous fuzzy differential equations, Fuzzy Sets and Systems, 102 (1999), $259-262$. 1

[25] M. L. Puri, D. A. Ralescu, Differentials of fuzzy functions, J. Math. Anal. Appl., 91 (1983), 552-558. 1, 2

[26] D. Qiu, W. Zhang, C.-X. Lu, On fuzzy differential equations in the quotient space of fuzzy numbers, Fuzzy Sets and Systems, 295 (2016), 72-98. 1

[27] S. Seikkala, On the fuzzy initial value problem, Fuzzy Sets and Systems, 24 (1987), 319-330. 1

[28] A. Sikorska-Nowak, Retarded functional differential equations in Banach spaces and Henstock-Kurzweil integrals, Demonstratio Math., 35 (2002),49-60. 4.1

[29] T.-L. Toh, T.-S. Chew, On functional-differential equations with unbounded delay and Henstock-Kurzweil integrals, New Zealand J. Math., 28 (1999), 111-123. 1

[30] N. Van Hoa, P. Van Tri, T. Trong Dao, I. Zelinka, Some global existence results and stability theorem for fuzzy functional differential equations, J. Intell. Fuzzy Systems, 28 (2015), 393-409. 1

[31] D.-G. Wang, W.-Y. Song, H.-X. Li, Approximation properties of ELM-fuzzy systems for smooth functions and their derivatives, Neurocomputing, 149 (2015), 265-274. 1

[32] C.-X. Wu, Z.-T. Gong, On Henstock integrals of interval-valued functions and fuzzy-valued functions, Fuzzy Sets and Systems, 115 (2000), 377-391. 1

[33] C.-X. Wu, Z.-T. Gong, On Henstock integral of fuzzy-number-valued functions, I, Fuzzy Sets and Systems, 120 (2001), 523-532. 1

[34] C.-X. Wu, M. Ma, Embedding problem of fuzzy number space, II, Fuzzy Sets and Systems, 45 (1992), 189-202. 2.1

[35] X.-P. Xue, Y.-Q. Fu, Carathéodory solutions of fuzzy differential equations, Fuzzy Sets and Systems, 125 (2002), $239-243$. $1,4.2$ 